DOI: $10.1515 / \mathrm{rrlm}-2017-0012$

\title{
Investigation of biomarker variations post-return of spontaneous circulation following an out-of-hospital cardiac arrest
}

\author{
Adela Golea ${ }^{1}$, Adriana Rusu ${ }^{*}$, Christiana Dumulesc ${ }^{3}$, Cornelia Bala ${ }^{2}$ \\ 1. Iuliu Hatieganu University of Medicine and Pharmacy, Department of Emergency Medicine, Cluj- \\ Napoca, Romania; 2. Iuliu Hatieganu University of Medicine and Pharmacy, Department of Diabetes, \\ Nutrition and Metabolic Diseases, Cluj-Napoca, Romania; 3. Iuliu Hatieganu \\ University of Medicine and Pharmacy, Cluj-Napoca, Romania
}

\begin{abstract}
Objective: The objective of this research was to describe evolution of several biomarkers post-return of spontaneous circulation (ROSC) following an out-of-hospital cardiac arrest (OHCA).

Methods: Thirteen adult patients were divided in 2 groups according to their survival status at 30 days, survivors (alive at 30 days or discharged alive) and non-survivors (not alive at 30 days). Glycemia, lactate, C-reactive protein (CRP), neurofilament heavy chain (NfH) and presepsin were assessed at pre-set time-points, during OHCA and the first 72 hours post-ROSC.

Results: In survivors, lactate levels decreased steadily throughout the 72 hours from a maximum observed during OHCA; in non-survivors, it increased during ROSC, then decreased abruptly at 2 hours post-ROSC and remained lower than in survivors for up to 24 hours. Glycemia at all-time points within the first 24 hours and CRP levels at 2 hours post-ROSC were higher in non-survivors, but this observed difference was not statistically significant. The variation of NfH was bi-modal, with peaks at 12 and 48 hours. The interpretation of NfH was limited by the large number of samples outside the limit of detection.

Conclusion: Glycemia, lactate and CRP showed different patterns of evolution in survivors and non-survivors and should be further investigated as potential predictors of survival after ROSC.

Keywords: out of hospital cardiac arrest, return of spontaneous circulation, biomarkers

Received: 11 ${ }^{\text {th }}$ December 2016; Accepted: $6^{\text {th }}$ March 2017; Published: $16^{\text {th }}$ May 2017

\section{Introduction}

Out-of-hospital cardiac arrest (OHCA) is one of the major causes of death in Europe and USA. Survival after cardiac arrest and survival with good neurological function remains a ma-

jor public healthcare problem and prediction of survival and especially survival with good neurological function remains a huge unmet need of the healthcare systems. Post cardiac arrest syndrome, consisting of anoxic brain injury,
\end{abstract}

\footnotetext{
*Corresponding author: Adriana Rusu, Iuliu Hatieganu University of Medicine and Pharmacy, Department of Diabetes, Nutrition and Metabolic Diseases, Romania, e-mail: adi.rusu@yahoo.com
} 
myocardial dysfunction, and systemic ischemia/ reperfusion response following the return of spontaneous circulation (ROSC) after complete whole-body ischemia, remains a major cause of mortality with little changes in survival during the past 50 years despite major advances in the treatment of cardiac arrest [7].

Several biomarkers, such as serum lactate, C-reactive protein (CRP) and neurofilament heavy chain $(\mathrm{NfH})$ have been proposed as predictors of survival following ROSC. However, research results have been mixed and with questionable applicability in the real-life settings in terms of risk stratification after ROSC. Lactate level has been shown to be associated with mortality in trauma, burns, sepsis and ROSC after cardiac arrest [8-11]. NfH belongs to the intermediate filaments class, are abundant in axons and are present in both central and peripheral nervous system, playing a central role in axon growing [12]. Increased serum levels of $\mathrm{NfH}$ have been reported in states associated with neuronal injury [13-14], and it has been proposed as surrogate markers of brain injury after cardiac arrest [15]. Post-cardiac arrest immune-inflammatory response, especially the level of CRP, has been correlated with patients' survival following cardiac arrest [16]. The levels of N-terminal fragment of the soluble CD14 form, known as presepsin, produced by hepatocytes and involved in the immune responses of endothelial and epithelial cells [17] increases in the serum during the inflammatory stress, and has been proposed as a serum biomarker for early diagnosis of sepsis [17, 18] but its value as an inflammatory biomarker following ROSC has yet to be established.

We hypothesised that in patients with ROSC following cardiac arrest, the levels of several biomarkers such as lactate, glycemia, CRP, NfH, and presepsin may display rapid changes within the first hours and days that are not yet fully de- scribed and that these fluctuations can have an impact on their ability to predict outcomes.

\section{Objective}

The main objective of the research presented here was to describe the variation in the first 72 hours post-ROSC of lactate, glycemia, CRP, $\mathrm{NfH}$, and presepsin according to patients' survival status at 30 days or discharge.

\section{Materials and method}

\section{Study design and patients}

This was a prospective observational research conducted in the Prehospital Intensive Care Units and Emergency Department of the Emergency Clinical County Hospital Cluj between July 2014 and April 2015. Adult patients with resuscitated non-traumatic OHCA, with ventricular fibrillation as initial arrest rhythm at the ambulance presentation or during the resuscitation, and for whom the family members consented for data collection and blood sampling were included in the study. Patients with oncological medical history, and patients who did not achieve a return of spontaneous circulation in pre-hospital setting were excluded from the study.

Eligible patients were followed until discharge or death, whichever came first. Patients were divided in 2 groups for the analysis: survivors (patients who were alive at 30 days and patients discharged alive) and non-survivors (patients who were not alive at 30 days after ROSC). Pre-hospital treatment and treatment in the Emergency Department and subsequently in the Intensive Care Unit (ICU) was performed according to the Emergency Clinical County Hospital Cluj procedures.

The research was approved by the Ethics Committee of the "Iuliu Haţieganu" University of Medicine and Pharmacy Cluj-Napoca, Ro- 
mania (No.107/28.02.2014) and conducted in accordance with the International Conference on Harmonization Good Clinical Practice guidelines and the Declaration of Helsinki, as revised in 2000. One of the family members signed the informed consent for the participation to the study.

\section{Study assessments}

Data on age, sex, medical history, initial arrest rhythm, time from dispatch call to start of advanced life support maneuvres (time to ALS), the presence or absence of bystander CPR, number of cardiac resuscitation attempts, adrenaline dose (mg), systolic and diastolic blood pressure at ROSC and number of days of admittance were collected from the Prehospital Intensive Care Units and Emergency Department records.

Glucose, lactate, CRP, presepsin, and $\mathrm{NfH}$ were collected at pre-set time-points, as per protocol, before and after the ROSC. Samples for glucose and lactate were collected during the cardiac arrest, at ROSC, at admittance in the Emergency Department, and at 2, 6, 12, 24, 36, 48 and 72 hours following the ROSC. Samples for CRP and presepsin were obtained before admittance in the Emergency Department, and at 2, 6 and 12 hours following the ROSC and those for $\mathrm{NfH}$ were collected at 2, 6, 12, 24, 36, 48 and 72 hours following ROSC.

Routine enzymatic methods (Konelab 30, Thermo Fisher Scientific Inc, Finland) were used for the assessments of plasma glucose levels. Presepsin and CRP were measured from whole blood samples on PATHFAST ${ }^{\mathrm{TM}}$ analyzer (Mitsubishi Chemical Holdings Corporation, Tokyo, Japan) by PATHFAST ${ }^{\mathrm{TM}}$ Presepsin chemiluminescent enzyme immunoassay (Mitsubishi Chemical Holdings Corporation, Tokyo, Japan), assay range $20-20000 \mathrm{pg} / \mathrm{mL}$ and by PATHFAST ${ }^{\circledR}$ hsCRP enzyme immunoassay (Mitsubishi Chemical Holdings Corporation, Tokyo, Japan), assay range 0.05-30 mg/dL. Lactate and arterial $\mathrm{pH}$ were determined by routine methods using RAPIDPoint ${ }^{\circledR} 500$ Blood Gas Systems (Siemens Healthcare GmbH, Erlangen, Germany).

The samples for $\mathrm{NfH}$ assessment were immediately centrifuged to separate the blood cells, frozen and stored at $-70^{\circ} \mathrm{C}$ until the analysis. The assessments were performed using commercially available kits (MyBioSource.com, San Diego, CA, United States) using a standard ELISA assay. Assay ranges: 15.6-1000pg/mL.

\section{Statistical analysis}

Descriptive statistic (mean, standard deviation, median, quartile 1 and quartile 3) were computed for continuous variables. Categorical variables were summarized with frequency tables (number and percentage). Student t-test, independent samples median test and chi-square test were used to compare variables between the survivors and non-survivors groups. A p value below 0.05 was considered statistically significant. For the statistical analysis, the CRP and $\mathrm{NfH}$ values below or above the limits of quantification of the tests were not included in the analyses of medians and are presented separately.

Statistical analysis was carried out using IBM $^{\circledR}$ SPSS $^{\circledR}$ Statistics (IBM, Armonk, NY, USA).

\section{Results}

Of the 46 cases which fulfilled the inclusion criteria and without any exclusion criteria, blood samples were obtained from 13 patients. Overall, 7 of the 13 patients (53.8\%) survived up to 30 days or to discharge (survivors) and 6 patients (46.2\%) died before discharge (non-survivors). The demographic and clinical characteristics of the survivors and non-survivors group are presented in Table I.

Overall, serum lactate had the highest levels pre-hospital during the cardiac arrest (median value $11.5 \mathrm{mmol} / \mathrm{L}$ ), displaying a decreasing trend up to 6 hours post cardiac arrest (median value $2.5 \mathrm{mmol} / \mathrm{L}$ ) and plateaued thereafter up 
Table 1. Baseline characteristics of the patients

\begin{tabular}{|c|c|c|c|c|}
\hline Parameter & $\begin{array}{l}\text { Total } \\
\mathrm{N}=13\end{array}$ & $\begin{array}{l}\text { Survivors } \\
\quad \mathrm{N}=7\end{array}$ & $\begin{array}{c}\text { Non-survivors } \\
\quad \mathrm{N}=6\end{array}$ & p-value \\
\hline Age, years & $59.9(18.6)$ & $52.83(20.23)$ & $68.40(13.85)$ & 0.180 \\
\hline Men, n (\%) & $9(69.2 \%)$ & $6(85.7 \%)$ & $3(50.0 \%)$ & 0.164 \\
\hline CVD history, $\mathrm{n}(\%)$ & $7(53.8 \%)$ & $4(57.1 \%)$ & $3(50.0 \%)$ & 0.967 \\
\hline $\begin{array}{l}\text { Initial arrest rhythm, n (\%) } \\
\text { - PEA } \\
\text { - Asystole } \\
\text { - VF }\end{array}$ & $\begin{array}{c}1(7.7) \\
3(23.1) \\
9(69.2)\end{array}$ & $\begin{array}{c}0(0 \%) \\
3(42.9 \%) \\
4(57.1 \%)\end{array}$ & $\begin{array}{c}1(16.7 \%) \\
0(0 \%) \\
5(83.3 \%)\end{array}$ & 0.131 \\
\hline Presence of bystander CPR, n (\%) & $6(46.2)$ & $3(42.9 \%)$ & $3(50.0 \%)$ & 0.797 \\
\hline Time to ALS (min), n (Q1; Q3) & $5.0(4.0 ; 6.5)$ & $5.0(4.0 ; 5.0)$ & $5.0(4.0 ; 8.0)$ & 1.000 \\
\hline $\begin{array}{l}\text { Number of resuscitation attempts, } \mathrm{n} \\
\text { (Q1; Q3) }\end{array}$ & $\begin{array}{c}3.00 \\
(2.00 ; 4.00)\end{array}$ & $\begin{array}{c}2.00 \\
(1.50 ; 4.00)\end{array}$ & $\begin{array}{c}3.50 \\
(3.00 ; 4.00)\end{array}$ & 0.592 \\
\hline Adrenalin dose, $\mathrm{mg}$ & $6.4(3.7)$ & $5.71(3.68)$ & $7.40(3.78)$ & 0.457 \\
\hline $\mathrm{SBP}, \mathrm{mmHg}$ & $138.2(48.2)$ & $121.29(50.71)$ & $158.00(40.89)$ & 0.184 \\
\hline DBP, mmHg & $90.9(27.4)$ & $84.71(26.66)$ & $98.17(28.90)$ & 0.401 \\
\hline
\end{tabular}

All continuous variables results are displayed as mean (standard deviation) if not specified otherwise.

$\mathrm{n}(\%)$, number (percentage); PEA, pulseless electrical activity; VF, ventricular fibrillation; CVD, cardiovascular disease; SBP, systolic blood pressure; DBP, diastolic blood pressure

to 24 hours (median value $2.3 \mathrm{mmol} / \mathrm{L}$; Figure 1A). After 24 hours the serum lactate started to decrease reaching its minimum values at 48 and 72 hours post-ROSC $(1.6 \mathrm{mmol} / \mathrm{L})$. The pattern of serum lactate levels observed during the first 36 hours was different in survivors than in non-survivors. In survivors, the maximum lactate value was recorded in the first pre-hospital sample (median value $12.1 \mathrm{mmol} / \mathrm{L}$ ) and the lactate decreased steadily up to $2.0 \mathrm{mmol} / \mathrm{L}$ at 36 hours. In non-survivors the median lactate levels increased from $8.0 \mathrm{mmol} / \mathrm{L}$ in the first pre-hospital sample to $9.4 \mathrm{mmol} / \mathrm{L}$ at admittance, started to decrease following the admittance reaching $1.6 \mathrm{mmol} / \mathrm{L}$ at 24 hours. The difference observed between the serum lactate levels in the 2 study groups was not statistically significant at any timepoint ( $p>0.05$ for all).

Glucose median values increased from 91.0 $\mathrm{mg} / \mathrm{dL}$ during the cardiac arrest to $225.5 \mathrm{mg} / \mathrm{dL}$ after RCOS and $281.0 \mathrm{mg} / \mathrm{dL}$ at admittance and then steadily decreased up to 36 hours (median value $127.5 \mathrm{mg} / \mathrm{dL}$ ) and plateaued thereafter up to 72 hours. The trend and the levels was similar in both survivals and non-survivals ( $p>0.05$ for all; Figure 1B).

In the whole population analysed CRP median values decreased from $6.7 \mathrm{mg} / \mathrm{L}$ pre-hospital admittance to $2.5 \mathrm{mg} / \mathrm{L}$ at 2 hours after ROSC and then started to increase. The increasing trend was maintained at 12 hours postROSC. A similar trend was observed in the survivors. In non-survivors, the CRP values increased steadily from $3 \mathrm{mg} / \mathrm{dL}$ pre-hospital and reached the maximum level at 12 hours (14.7 $\mathrm{mg} / \mathrm{L}$; Figure 1C).

Median presepsin levels increased from pre-hospital $(500.0 \mathrm{pg} / \mathrm{mL})$ reaching its peak at 2 hours post-ROSC $(889.0 \mathrm{pg} / \mathrm{mL})$ and then decreased to $483.0 \mathrm{pg} / \mathrm{mL}$ at 6 hours post ROSC. In survivors presepsin levels remained relatively stable and high from 2 to 12 hours post-ROSC; in non-survivors presepsin levels started to decrease after 2 hours post-ROSC and maintained the decreasing trend at 12 hours post-ROSC. At all-time points presepsin levels were higher in 
$\mathbf{A}$



B

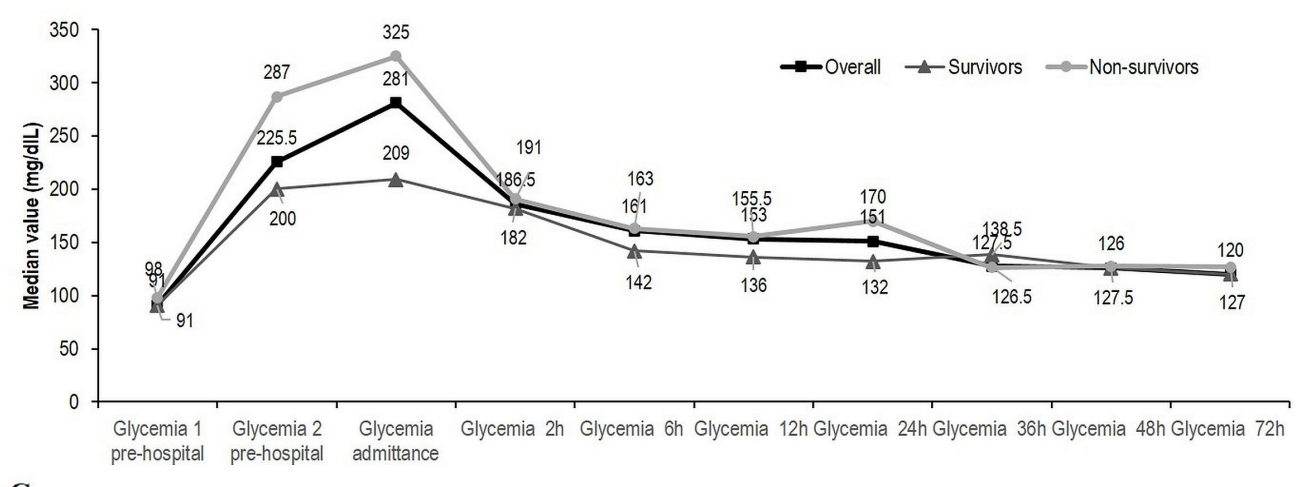

C

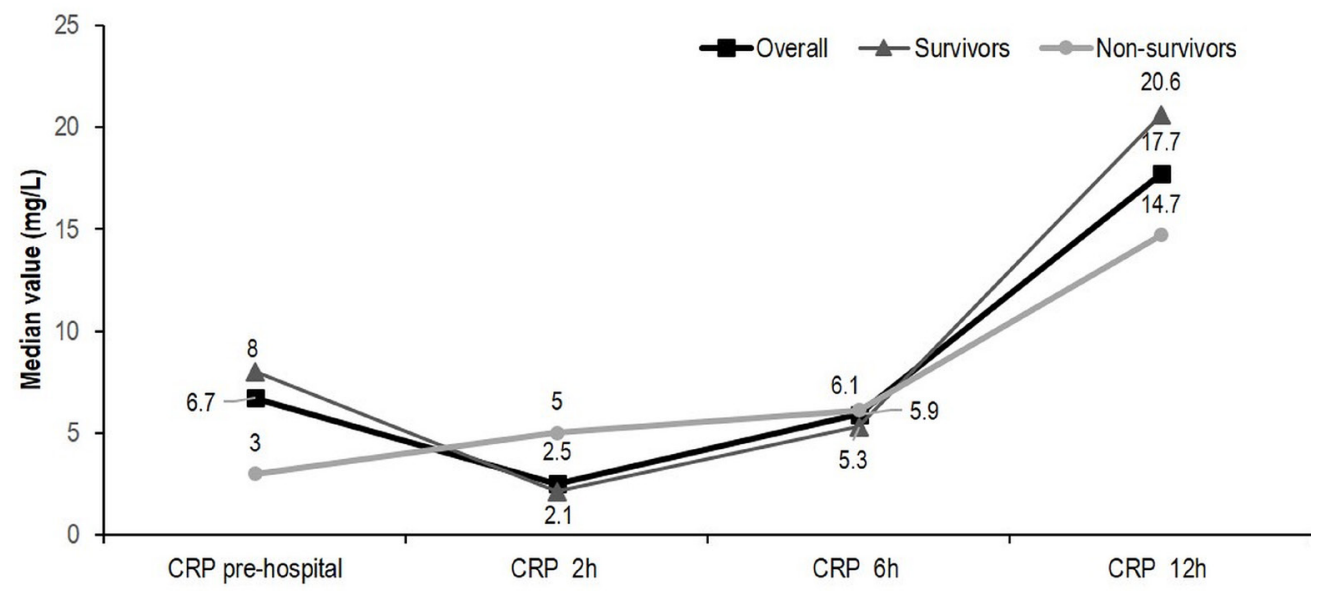

Figure 1. Evolution of median lactate (panel A), glycemia (panel B) and CRP (panel C) levels in the whole group included in the analysis and separate in survivors and non-survivors

lactate 1 pre-hospital, lactate collected during cardiac arrest; lactate 2 pre-hospital, lactate collected at ROSC; glycemia 1 pre-hospital, glycemia collected during cardiac arrest; glycemia 2 pre-hospital, glycemia collected at ROSC; ROSC, return of spontaneous circulation; CRP, C-reactive protein 


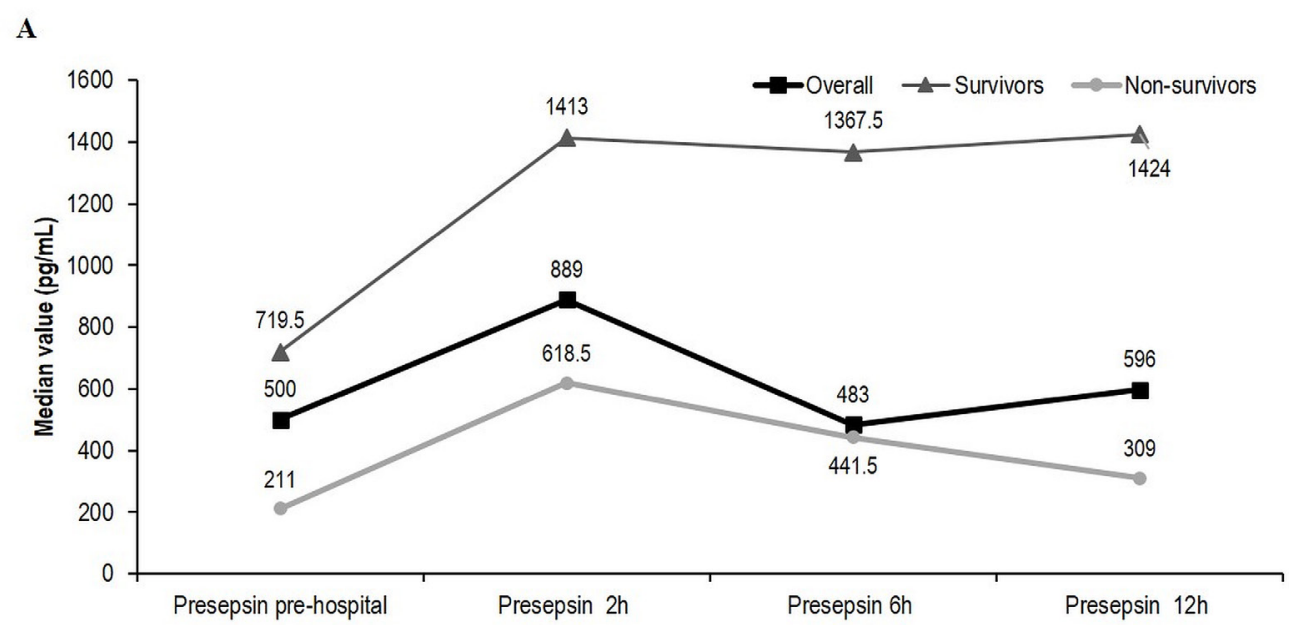

B

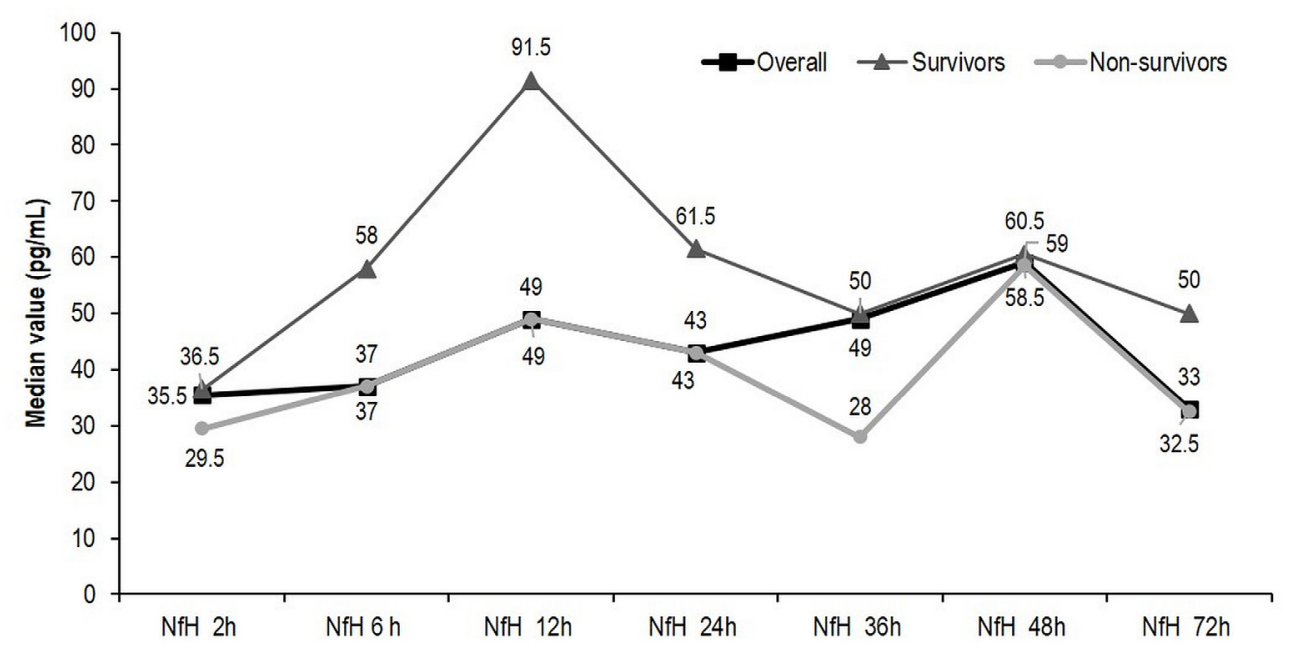

Figure 2. Evolution of median presepsin (panel A) and neurofilament heavy chain (panel B) in the whole group included in the analysis and separate in survivors and non-survivors

$\mathrm{NfH}$, neurofilament heavy chain

survivors than in non-survivors, but the differences did not reach statistical significance $(\mathrm{p}$ $>0.05$; Figure 2A).

Overall, median $\mathrm{NfH}$ values ranged between $33.0 \mathrm{pg} / \mathrm{mL}$ and $59.0 \mathrm{pg} / \mathrm{mL}$. During the time interval 2-72 hours post-ROSC NfH had a bimodal evolution with the minimum values observed at 2 and 72 hours post-ROSC and 2 peaks - one at 12 hours post-ROSC and one at 48 hours post ROSC. This bimodal evolution of the median
$\mathrm{NfH}$ levels was observed in both survivors and non-survivors, who had similar NfH levels at all timepoints ( $p>0.05$ for all; Figure 2B).

CRP levels exceed the limit of quantification of the test of $30 \mathrm{mg} / \mathrm{L}$ at all time points in 2 patients from the survivors group and at 12 hours in 3 patients from the non-survivors group. Of the 80 available $\mathrm{NfH}$ samples for all patients, 13 were below and 3 above the limit of quantification for the test. 


\section{Discussion}

We performed a study which aimed to describe the short-term fluctuations of a number of biomarkers within the first 72 hours post-ROSC in non-traumatic OHCA patients according to their survival status at 30 days or discharged alive from hospital.

In post-cardiac arrest patients, lactic acidosis may occur due to increased lactate production as a consequence of microcirculatory and mitochondrial dysfunction leading to inadequate tissue perfusion and oxygen supply or decreased lactate clearance $[11,19]$. In a retrospective study including 128 patients with ROSC following OHCA, it was shown that mortality increased in parallel with serum lactate levels collected within one hour from ROSC, from $39 \%$ in those with a lactate level $<5 \mathrm{mmol} / \mathrm{L}$ to $92 \%$ in those with lactate level $\geq 10 \mathrm{mmol} / \mathrm{L}$ [11]. We did not observe any statistically significant difference between the lactate levels in the 2 study groups. These results may be explained by various etiologies of cardiac arrest or comorbidities known to be associated with high lactate levels, such as hepatic failure or thiamine deficiency, and which were not evaluated. Also lactate levels are influenced by the duration of the cardiac arrest [20]. We were not able to accurately collect data on the duration of cardiac arrest and the only variable available was the time to ALS, which was similar in survivors and non-survivors. Our data showed that in the survivors' group lactate levels decreased steadily throughout the 72 hours following cardiac arrest. In non-survivors, lactate increased during ROSC and then decreased abruptly at 2 hours following ROSC. The pattern observed in the non-survivors' group may suggest that rapid fluctuations in lactate levels within the first 2 hours following ROSC are critical in establishing a relationship between lactate levels and survival at 30 days.

Numerous studies have shown that hyperglycemia is an important outcome predictor in crit- ically ill patients with or without diabetes. In a prospective study on 1,000 consecutive patients admitted in an intensive care unit, each $1 \mathrm{mmol} / 1$ increase in peak blood glucose during the first 48 hours was associated with a $20 \%$ increase in risk of death in patients with critical illness-associated hyperglycemia and in those with established diabetes and $\mathrm{HbA} 1 \mathrm{c}$ levels $<7 \%$ [21]. In a group of 134 patients who survived to hospital admission after an OHCA, in those who were alive at discharge blood glucose levels remained stable between pre-hospital and admission, while in non-survivors there was a significant increase between these 2 time points [22]. We also observed that survivors had lower levels of blood glucose than non-survivors, although the difference did not reach statistical significance.

In patients with successfully resuscitated cardiac arrest, a "systemic inflammatory response syndrome" was described in the first hours following resuscitation [23]. Higher levels of inflammatory cytokines on admission were seen in non-survivors as compared to survivors [23]. We observed higher CRP levels at 6 and 12 hours in survivors than in non-survivors, although this difference was not statistically different. No studies reporting levels of presepsin in patients with ROSC following cardiac arrest were found in the literature. In our study, pre-hospital presepsin values in the whole group were $500 \mathrm{pg} / \mathrm{mL}$, similar to levels seen in a group of patients with cardiogenic shock with or without infectious complications [18] and a rapid increase of presepsin was observed at 2 hours for both survivors and non-survivors. We did not collect patient diagnosis and therefore we cannot exclude the presence of sepsis and its association with higher presepsin levels in the whole sample and higher CRP levels in survivors.

We found a bi-modal variation of $\mathrm{NfH}$, with peaks at 12 and 48 hours for $\mathrm{NfH}$. A similar pattern with latter peaks of $\mathrm{NfH}$ values ( $>24$ hours) was reported in a study on 90 patients treated 
with hypothermia after cardiac arrest [15]. The interpretation of NfH levels in our study is limited by the large number of samples outside the limit of detection (16 out of 80 samples). If for those above the limit of quantification the dilution should be used, we had several samples below the limit of detection of the assay thus limiting its utility in patients post-ROSC. The same limitation in the assay ranges for commercially available kits was reported in the study of Rundgren et al [15], where for a large proportion of patients were reported $\mathrm{NfH}$ levels of $0 \mathrm{pg} / \mathrm{mL}$, with the value of 0 attributed to non-measurable levels.

Several limitations of our study should be acknowledged. The main limitation was the small number of patients evaluated due to objective difficulties in evaluating cardiac arrest patients during CPR and immediately after ROSC. Thus, the statistical comparison of between group differences may have not been accurate and of limited interpretability. Although no statistically significant difference between study groups was observed in terms of age, the survivors were 16 years younger than non-survivors and this may had influenced the survival and thus also the pattern of studied markers.

\section{Conclusions}

Although no statistically significant difference was observed in our patients, glycemia, lactate and CRP levels seem to display different patterns of evolution in survivors and non-survivors following ROSC after cardiac arrest. Studies including larger samples, allowing for adjustment for other potential cofounders known to influence the survival and including clinical parameters are needed to further investigate the evolution of these markers as potential predictors of survival after ROSC.

\section{Abbreviations}

ALS - advanced life support; CRP - C-reactive protein; $\mathrm{NfH}$ - neurofilament heavy chain;
ICU - Intensive Care Unit; OHCA - out-of-hospital cardiac arrest; ROSC - return of spontaneous circulation

\section{Acknowledgement}

This study was supported by a grant financed by "Iuliu Haţieganu" University of Medicine and Pharmacy Cluj-Napoca, Romania (contract no.1493/19/28.01.2014); equipment used from Hospital and County Ambulance Service according to the university ethical approval of the study (No.107/28.02.2014).

\section{Conflict of interest}

Authors have no conflicts of interest to declare.

\section{References}

1. Mozaffarian D, Benjamin EJ, Go AS, Arnett DK, Blaha MJ, Cushman M, et al. Heart disease and stroke statistics--2015 update: a report from the American Heart Association. Circulation. 2015 Jan;131(4):e29-322. DOI: 10.1161/CIR.0000000000000152

2. Muller D, Agrawal R, Arntz HR. How sudden is sudden cardiac death? Circulation. Sep;114(11):1146-50.

3. Nichol G, Thomas E, Callaway CW, Hedges J, Powell JL, Aufderheide TP, et al. Regional variation in out-of-hospital cardiac arrest incidence and outcome. JAMA. Sep;300(12):1423-31.

4. Centers for Disease Control and Prevention. 2013 Cardiac Arrest Registry to Enhance Survival (CARES) National Summary Report. https://mycares.net/sitepages/ uploads/2014/2013CARESNationalSummaryReport. pdf. Accessed November 11, 2016.

5. Gräsner JT, Böttiger BW, Bossaert L; European Registry of Cardiac Arrest (EuReCa) ONE Steering Committee; EuReCa ONE Study Management Team. EuReCa ONE - ONE month - ONE Europe - ONE goal. Resuscitation. 2014 Oct;85(10):1307-8. DOI: 10.1016/j. resuscitation.2014.08.001

6. Gräsner JT, Lefering R, Koster RW, Masterson S, Böttiger BW, Herlitz J, et al. EuReCa ONE-27 Nations, ONE Europe, ONE Registry: A prospective one month analysis of out-of-hospital cardiac arrest outcomes in 27 
countries in Europe. Resuscitation. 2016 Aug;105:18895. DOI: 10.1016/j.resuscitation.2016.06.004

7. Neumar RW, Nolan JP, Adrie C, Aibiki M, Berg RA, Böttiger BW, et al. Post-cardiac arrest syndrome: epidemiology, pathophysiology, treatment, and prognostication. A consensus statement from the International Liaison Committee on Resuscitation (American Heart Association, Australian and New Zealand Council on Resuscitation, European Resuscitation Council, Heart and Stroke Foundation of Canada, InterAmerican Heart Foundation, Resuscitation Council of Asia, and the Resuscitation Council of Southern Africa); the American Heart Association Emergency Cardiovascular Care Committee; the Council on Cardiovascular Surgery and Anesthesia; the Council on Cardiopulmonary, Perioperative, and Critical Care; the Council on Clinical Cardiology; and the Stroke Council. Circulation. 2008 Dec;118(23):2452-83. DOI: 10.1161/CIRCULATIONAHA.108.190652

8. Jeng JC, Jablonski K, Bridgeman A, Jordan MH. Serum lactate, not base deficit, rapidly predicts survival after major burns. Burns 2002 Mar;28(2):161-6. DOI: 10.1016/S0305-4179(01)00098-5

9. Kamolz LP, Andel H, Schramm W, Meissl G, Herndon DN, Frey M. Lactate: early predictor of morbidity and mortality in patients with severe burns. Burns 2005 Dec;31(8):986-90. DOI: 10.1016/j.burns.2005.06.019

10. Callaway DW, Shapiro NI, Donnino MW, Baker C, Rosen CL. Serum lactate and base deficit as predictors of mortality in normotensive elderly blunt trauma patients. J Trauma 2009; Apr;66(4):1040-4. DOI: 10.1097/TA.0b013e3181895e9e

11. Cocchi MN, Miller J, Hunziker S, Carney E, Salciccioli J, Farris S. The association of lactate and vasopressor need for mortality prediction in survivors of cardiac arrest. Minerva Anestesiol. 2011 Nov;77(11):1063-71.

12. Yuan A, Rao MV, Veeranna, Nixon RA. Neurofilaments at a glance. J Cell Sci. 2012 Jul;125(Pt 14):3257-63. DOI: $10.1242 /$ jcs. 104729

13. Petzold A. Neurofilament phosphoforma: Surrogate markters for axomal injury, degeneration and loss. J Neurol Sci. 2005 Jun 15;233(1-2):183-98. DOI: 10.1016/j.jns.2005.03.015

14. Kuhle J, Leppert D, Petzold A, Regeniter A, Schindler C, Mehlin M, Anthony DC, Kappos L, Lindberg
RL. Neurofilament heavy chain in CSF correlates with relapses and disability in multiple sclerosis. Neurology. 2011 Apr 5;76(14):1206-13. DOI: 10.1212/ WNL.0b013e31821432ff

15. Rundgren M, Friberg H, Cronberg T, Romner B, Petzold A. Serial soluble neurofilament heavy chain in plasma as a marker of brain injury after cardiac arrest. Crit Care. 2012 Dec;16(2):R45. DOI: 10.1186/ cc11244

16. Samborska-Sablik A, Sablik Z, Gaszynski W. The role of the immuno-inflammatory response in patients after cardiac arrest. Arch Med Sci. 2011 Aug;7(4):619-26. DOI: 10.5114/aoms.2011.24131

17. Wu J, Hu L, Zhang G, Wu F, He T. Accuracy of Presepsin in Sepsis Diagnosis: A Systematic Review and Meta-Analysis. PLoS One. 2015 Jul;10(7):e0133057. DOI: 10.1371/journal.pone.0133057

18. Sandquist M, Wong HR. Biomarkers of sepsis and their potential value in diagnosis, prognosis and treatment. Clin Immunol. 2014 Oct;10(10):1349-56. DOI: 10.1586/1744666x.2014.949675

19. Donnino MW, Miller J, Goyal N, Loomba M, Sankey SS, Dolcourt B, et al. Effective lactate clearance is associated with improved outcome in post-cardiac arrest patients. Resuscitation. 2007 Nov;75(2):229-34. DOI: 10.1016/j.resuscitation.2007.03.021

20. Andersen LW, Mackenhauer J, Roberts JC, Berg KM, Cocchi MN, Donnino MW. Etiology and therapeutic approach to elevated lactate levels. Mayo Clin Proc. 2013 Oct;88(10):1127-40. DOI: 10.1016/j. mayocp.2013.06.012

21. Plummer MP, Bellomo R, Cousins CE, Annink CE, Sundararajan K, Reddi BA, et al. Dysglycaemia in the critically ill and the interaction of chronic and acute glycaemia with mortality. Intensive Care Med. 2014 Jul;40(7): 973-80. DOI: 10.1007/s00134-014-3287-7

22. Nurmi J, Boyd J, Anttalainen N, Westerbacka J, Kuisma M. Early increase in blood glucose in patients resuscitated from out-of-hospital ventricular fibrillation predicts poor outcome. Diabetes Care. 2012 Mar;35:5102. DOI: $10.2337 / \mathrm{dc} 11-1478$

23. Adrie C, Adib-Conquy M, Laurent I, Monchi M, Vinsonneau C, Fitting C, et al. Successful cardiopulmonary resuscitation after cardiac arrest as a sepsis-like syndrome. Circulation. 2002 Jul;106:562-8. DOI: 
10.1161/01.CIR.0000023891.80661.AD

24. Peberdy MA, Andersen LW, Abbate A, Thacker LR, Gaieski D, Abella B, et al. Inflammatory markers following resuscitation from out-of-hospital cardiac arrest-A prospective multicenter observational study. Resuscitation. 2016 Jun;103:117-24. DOI: 10.1016/j. resuscitation.2016.01.006

25. Zhang YP, Zhu YB, Duan DD, Fan XM, He Y, Su JW, et al. Serum UCH-L1 as a Novel Biomarker to Predict Neuronal Apoptosis Following Deep Hypothermic Circulatory Arrest. Int J Med Sci. 2015 Jul;12(7):576-82. DOI: $10.7150 /$ ijms.12111 